\title{
Editorial
}

\section{Preventing Transmission of Hepatitis B Virus From Surgeons to Patients}

\author{
Linda A. Chiarello, RN, MS; Denise M. Cardo, MD
}

Isolated episodes of transmission of hepatitis B virus (HBV), hepatitis $\mathrm{C}$ virus, and human immunodeficiency virus (HIV) from infected healthcare providers to patients in healthcare settings have been reported. Most HBV transmissions have occurred during invasive surgical or obstetric procedures. In general, three conditions are necessary for transmission of blood-borne viruses from healthcare personnel to patients: (1) the healthcare provider must be infected and have the virus circulating in the bloodstream; (2) the healthcare provider must be injured or have a condition that provides some other source of direct exposure to infected blood or body fluids; and (3) the injury mechanism or condition must present an opportunity for the healthcare provider's blood to directly contact a patient's mucous membranes, wound, or traumatized tissue (recontact). ${ }^{1}$

The article by Spijkerman et al. in this issue of Infection Control and Hospital Epidemiology adds important information concerning the risk of transmission of $\mathrm{HBV}$ from infected surgeons to patients. ${ }^{2}$ The authors conducted a retrospective cohort study of 1,564 patients operated on by an HBV-infected surgeon and a case-control study to identify risk factors for transmission. Several findings from this investigation are similar to those of other reports of transmission, namely the episode involved a hepatitis B e antigen ( $\mathrm{HBeAg}$ )-positive healthcare provider with a high viral load and risk factors for transmission included longer duration of surgery and more highly invasive procedures. However, this epidemiologic study suggests that even the simplest procedures may not be risk free. The authors raise questions concerning appropriate policies for infected healthcare providers that call for response and discussion.

Certain aspects of this investigation are of particular interest. The outbreak involved a general surgeon who per- formed a variety of surgical procedures (eg, sigmoid and ileocecal resection, mastectomy, aorta bifurcation prosthesis, and ligation and stripping of varicose veins) that, in the United States, are usually performed by surgical specialists. This surgeon reportedly performed an average of 61 surgical procedures per month. By his own admission, he sustained frequent puncture injuries and noted suturerelated lesions on his hands. He wore double gloves during major trauma operations but apparently not during major elective surgical operations. No information is provided on surgical techniques that may have contributed to patient exposures (eg, the use of fingers as a backstop or to guide suture needles or the use of hands rather than instruments for wound retraction or exploration). However, surgical complications, including wound infection, were more common in cases than in controls. Whether they are indicative of poor quality of care in general cannot be determined based on the information provided.

Much of the evidence presented in this study may have important implications for current policies regarding infected healthcare providers. In particular, it calls into question the use of procedure category as the sole criterion for determining whether an infected healthcare provider poses a risk of transmission to patients. In 1991, the Center for Disease Control (CDC) attempted to create a list of "exposure-prone procedures" based on evidence of previous HBV transmission during a given invasive procedure, observational studies, or both that showed an increased risk for injury of healthcare providers during the procedures. However, this and subsequent efforts to create a list of exposure-prone procedures were unsuccessful, due in part to a failure to consider other factors that may influence the risk of transmission, such as technical skill and use of preventive practices by the healthcare provider. The oppor- 
tunities for patient exposure that arose from the suboptimal surgical techniques described in this article may explain how transmissions occurred during procedures that were not considered exposure prone.

Ten years ago, a national policy debate over episodes of transmission of HIV from an infected dentist to his patients led to the CDC Recommendations for Preventing Transmission of Human Immunodeficiency Virus and Hepatitis B Virus to Patients During Exposure-Prone Invasive Procedures. ${ }^{3}$ Subsequently, each state adopted either the CDC policy recommendations or their "equivalent," as required by federal law. Most states opted to establish equivalent policies that vary in the degree of oversight and restriction of infected healthcare providers. Position statements of major professional and healthcare organizations developed during the 1990 s also differ but tend to be less restrictive than those of the CDC or the state health departments. Policies in other countries also vary (eg, the United Kingdom has the most restrictive policy of any developed nation) and recent articles attest to a continued lack of consensus on this subject.,5

Public health policies must be grounded in science. At the time the 1991 CDC recommendations were written, although much was known about HBV, the science concerning HIV was limited. Since then, several sources of information have shown that the risk of healthcare provider-to-patient transmission of blood-borne viruses is extremely low. ${ }^{1}$ Gostin recently noted, "Current national policy offers no discernible risk reduction for patients. . . . consequently a new national policy should focus on structural changes to make the healthcare workplace safer for both patients and healthcare workers rather than on identification and management of infected healthcare workers." We agree. The policy should emphasize existing strategies to improve the safety of surgery and promote their wide implementation in healthcare facilities, including freestanding centers.

Prevention of transmission of blood-borne virus from patient to healthcare provider and from healthcare provider to patient during surgery requires a comprehensive approach that includes administering hepatitis $B$ vaccine to healthcare personnel, viewing all blood as potentially infectious, using measures to reduce blood exposure, and having a surgical team committed to promoting and maintaining a safe work environment. Hepatitis B vaccination levels are high among younger surgeons and vaccination is strongly promoted on entry into health professions schools. However, many older surgeons have not been vaccinated and should be targeted for this primary prevention intervention. ${ }^{67}$ Healthcare providers who perform surgical and gynecologic procedures also have a responsibility to know their blood-borne virus serostatus and, if positive, to seek advice from an expert consultant regarding patient safety. The surgeon in this report was a nonresponder to hepatitis B vaccine and should have been tested for hepatitis B surface antigen following that determination.

Currently, there are several ways to reduce the risk of injury during surgical procedures, including less invasive surgical approaches (eg, laparoscopic techniques), alternatives to needles and other sharps (eg, adhesive tape, staples, or glue for wound closure and electrocautery instead of scalpels), and sharps with injury prevention features (eg, blunted suture needles). Safe work practices are especially important in this setting. Examples include using instruments rather than hands for retracting and exploring tissue; avoiding the simultaneous presence of the hands of two or more surgeons in the operative field; and using a neutral zone (eg, emesis basin or Mayo stand) for passing sharps rather than using a hand-to-hand approach. ${ }^{8}$

Gloves provide an important barrier between patient and provider. However, glove perforation is common and introduces a risk of blood exposure between patient and provider and also breaches aseptic integrity. The benefits of double gloving, glove reinforcement, and new glove materials in preventing disease transmission have not been proven. Nevertheless, because of the evidence on its ability to reduce blood exposure, routine double gloving during invasive surgical and obstetric procedures is encouraged. 8,9

It is equally important that all members of the operating team be committed to safety. A broad systems approach that includes the measures outlined above will protect patients and healthcare workers more effectively than policies focused solely on infected healthcare providers.

\section{REFERENCES}

1. Chiarello IA, Cardo DM, Panlilio A, Alter MJ, Gerberding JL. Risks and prevention of bloodborne virus transmission from infected healthcare providers. Seminars in Infection Control 2001:1:61-72.

2. Spijkerman UB, van Doorn LJ, Janssen MHW, et al. Transmission of hepatitis $B$ virus from a surgeon to his patients during high-risk and lowrisk surgical procedures during 4 years. Infect Control Hosp Epidemiol 2002;23:306-312.

3. Center for Disease Control. Recommendations for preventing transmission of human immunodeficiency virus and hepatitis $B$ virus to patients during exposure-prone invasive procedures. $M M W R$ 1991;40(RR-8): $1-9$.

4. Gostin IO. A proposed national policy on health care workers living with HIV/AIDS and other blood-borne pathogens. JAMA 2000;284:1965-1970.

5. Ristinen E, Mamtani R. Ethics of transmission of hepatitis $B$ virus by health-care workers. Lancet 1998;352:1381-1383.

6. Panlilio AL, Shapiro CN, Schable CA, et al. Serosurvey of human immunodeficiency virus, hepatitis $B$ virus, and hepatitis $C$ virus among hospital-based surgeons. J Am Coll Surg 1995;180:16-24.

7. Shapiro $\mathrm{CN}$, Tokars JL, Chamberland ME. Use of the hepatitis-B vaccine and infection with hepatitis $\mathrm{B}$ and $\mathrm{C}$ among orthopaedic surgeons. $J$ Bone Joint Surg Am 1996;78:1791-1800.

8. Davis MS. Advanced Precautions for Today's OR: The Operating Room Professional's Handbook for the Prevention of Sharps Injuries and Bloodborne Exposures. Atlanta, GA: Sweinbinder Publications, LLC; 1999.

9. Gerberding JL, Quebbeman EK, Rhodes RS. Hand protection. Surg Clin North Am 1995;75:1133-1139. 